\title{
COMBINATORIAL IDENTITIES BY WAY OF WILF'S MULTIGRAPH MODEL
}

THERESA L. FRIEDMAN AND PAUL KLINGSBERG

Received 4 February 2005; Revised 29 October 2006; Accepted 9 November 2006

For many families of combinatorial objects, a construction of Wilf (1977) allows the members of the family to be viewed as paths in a directed multigraph. Introducing a partition of these paths generates a number of known, but hitherto disparate, combinatorial identities. We include several examples.

Copyright (c) 2006 Hindawi Publishing Corporation. All rights reserved.

\section{Introduction}

We begin with a well-known pair of identities, one satisfied by each type of Stirling number. For $1 \leq t \leq n$, Stirling numbers of the first kind are defined combinatorially by

$$
\left[\begin{array}{l}
n \\
t
\end{array}\right]:=\text { The number of } t \text {-cycle permutations of }[n] \text {. }
$$

For $1 \leq t<n$, these numbers satisfy the identity

$$
\left[\begin{array}{c}
n \\
t
\end{array}\right]=\sum_{1 \leq u_{1}<u_{2}<\cdots<u_{n-t} \leq n-1} u_{1} u_{2} \cdots u_{n-t} .
$$

For example,

$$
35=\left[\begin{array}{l}
5 \\
3
\end{array}\right]=1 \cdot 2+1 \cdot 3+1 \cdot 4+2 \cdot 3+2 \cdot 4+3 \cdot 4
$$

For $1 \leq t \leq n$, Stirling numbers of the second kind are defined by

$$
\left\{\begin{array}{l}
n \\
t
\end{array}\right\}:=\text { The number of } t \text {-block partitions of }[n] \text {. }
$$


For $1 \leq t<n$, these numbers satisfy the identity

$$
\left\{\begin{array}{c}
n \\
t
\end{array}\right\}=\sum_{1 \leq u_{1} \leq u_{2} \leq \cdots \leq u_{n-t} \leq t} u_{1} u_{2} \cdots u_{n-t}
$$

An example of this second identity is given by

$$
25=\left\{\begin{array}{l}
5 \\
3
\end{array}\right\}=1 \cdot 1+1 \cdot 2+1 \cdot 3+2 \cdot 2+2 \cdot 3+3 \cdot 3 .
$$

Combinatorial proofs of these identities can be found in [6], but we submit that the proper setting for understanding these identities as well as several others is one discovered by Wilf. In [7], Wilf provides a way to associate a directed multigraph to any family of combinatorial objects, provided only that the family admits a method of constructing the larger objects from the smaller ones. The set of all $t$-cycle permutations of $[n]$ (for all $t$ and $n$ ) constitutes such a family, as does the set of all $t$-block partitions of $[n]$; these are among the examples that Wilf gives in his paper. In this construction, the multigraph models the entire family, while individual objects correspond to certain paths in the graph; it turns out that partitioning the paths by vertex sequence generates a number of identities, including (1.2) and (1.5).

The formula that results from the partitioning unifies all of the identities we consider in this paper. Section 2 consists of a brief account of Wilf's construction and the most general version of our formula. In Sections 3 and 4, we specialize to contexts in which the multigraphs come with additional structure. In each of these two sections, the general formula assumes a more computational form, which then leads directly to a number of combinatorial identities. We discuss several examples, including (1.2) and (1.5). Finally, in Section 5, we discuss one identity that falls within the scope of the general formula but outside that of Sections 3 and 4.

\section{Combinatorial family graphs}

Definition 2.1. A combinatorial family graph $G$ is a directed multigraph on a (usually infinite) vertex set $V=V(G)$ satisfying the following conditions.

(a) Every vertex has finite indegree and finite outdegree.

(b) There is a unique vertex $\tau \in V$ (the "terminal vertex") of outdegree zero; for every other vertex $v$, the outdegree of $v, \rho_{\mathrm{o}}(v)$, is positive.

(c) $G$ has no paths of infinite length (so, in particular, $G$ is acyclic).

(d) For each $v \in V$, except for $\tau$, the edges with initial vertex $v$ are consecutively numbered $\left(1, \ldots, \rho_{\mathrm{o}}(v)\right)$.

Wilf's numbering of the edges differs slightly from ours, but in all other respects, this definition is that found in [7].

From (a) and (c), it follows that only finitely many paths originate from any fixed vertex. For any vertex $v$, let $b(v)$ denote the number of paths from $v$ to $\tau(b(\tau)=1)$; these paths are the combinatorial objects of order $v$. 
Since $G$ is a multigraph, there will generally be multiple paths that follow the same vertex sequence. Partitioning the set of all paths $v \rightarrow \tau$ by vertex sequence then gives our first theorem.

Theorem 2.2. For each $v \neq \tau$,

$$
b(v)=\sum_{\substack{\text { vertex } \\ \text { sequences }}}(\text { number of paths } v \longrightarrow \tau \text { with that vertex sequence). }
$$

\section{Application to restricted binomial families}

Most of the instances of combinatorial family graphs that we treat here belong to a subclass of combinatorial family graphs; in this subclass, we get useful special forms of (2.1). The following definition of a binomial family is essentially the one in [7], although there are a few minor differences.

Definition 3.1. A binomial family is a combinatorial family graph for which the following hold.

(a) For some $t_{0} \geq 0, V$ is a set of lattice points in the plane, namely, $V=\{(n, t): n \geq$ $\left.t \geq t_{0}\right\}$. The terminal vertex is $\tau=\left(t_{0}, t_{0}\right)$.

(b) For $n \geq(t+1) \geq\left(t_{0}+1\right)$, there will be $\phi(n, t) \geq 1$ horizontal edges $(n, t) \rightarrow$ $(n-1, t)$, numbered $(1,2, \ldots, \phi(n, t))$.

(c) For $n \geq t \geq\left(t_{0}+1\right)$, there will be $\psi(n, t) \geq 1$ diagonal edges $(n, t) \rightarrow(n-1, t-1)$, numbered $(\phi(n, t)+1, \ldots, \phi(n, t)+\psi(n, t))$.

In this section, we consider the simplest case, in which $\psi(n, t)=1$ for all $n \geq t \geq$ $\left(t_{0}+1\right)$. We call such binomial families restricted.

We now establish a computational form of (2.1) for restricted binomial families. For a vertex $(n, t)$ in a restricted binomial family $G$, fix one vertex sequence from $(n, t)$ to $\tau=\left(t_{0}, t_{0}\right)$. Such a sequence contains a total of $\left(n-t_{0}\right)$ moves, with $\left(t-t_{0}\right)$ of them being diagonal and $(n-t)$ of them being horizontal. The sequence can thus be indexed by the set

$$
S=\left\{k_{1}, k_{2}, \ldots, k_{n-t}\right\}
$$

of $x$-coordinates of vertices that occur to the right of the horizontal edges.

For example, the vertex sequence

$$
(0,0) \longleftarrow(1,1) \longleftarrow(2,1) \longleftarrow(3,2) \longleftarrow(4,2) \longleftarrow(5,2) \longleftarrow(6,3) \longleftarrow(7,3)
$$

will be indexed by the set $S=\{2,4,5,7\}$.

The set $S$ can now be used to find the number of paths with this vertex sequence. Since $\psi(x, y)=1$ for all $x \geq y \geq\left(t_{0}+1\right)$, the number of paths which follow this vertex sequence 
4 Combinatorial identities by way of Wilf's multigraph model

is given by

$$
\prod_{i=1}^{n-t} \phi\left(k_{i}, t_{i}\right)
$$

where $\left(k_{i}, t_{i}\right)$ is the vertex in the sequence with $x$-coordinate $k_{i}$. (Note that there is one path when $n=t$. Here and below, " 1 " is the correct value for any empty product.)

Furthermore, if we assume that the elements of $S$ have been arranged in increasing order $\left(k_{1}<k_{2}<\cdots<k_{n-t}\right)$, it is easy to see that $k_{i}=t_{i}+i$, so that

$$
\prod_{i=1}^{n-t} \phi\left(k_{i}, t_{i}\right)=\prod_{i=1}^{n-t} \phi\left(k_{i}, k_{i}-i\right) .
$$

Summing this expression over all possible vertex sequences from $(n, t)$ to $\left(t_{0}, t_{0}\right)$ then gives the following theorem.

Theorem 3.2. Let $G$ be a restricted binomial family, and let $(n, t) \in V(G)$, with $n \geq t+1$. Then,

$$
b(n, t)=\sum_{t_{0}+1 \leq k_{1}<k_{2}<\cdots<k_{n-t} \leq n}\left[\prod_{i=1}^{n-t} \phi\left(k_{i}, k_{i}-i\right)\right] .
$$

Note that $b(n, n)=1$.

Identities (1.2) and (1.5) are the instances of Theorem 3.2 that hold in the restricted binomial family of permutations (resp., partitions). These families appear in [7], but for completeness, we include them here.

Example 3.3. Let $V=\{(n, t): n \geq t \geq 1\}$; if $n \geq t+1 \geq 2$, let $\phi(n, t)=(n-1)$; if $n \geq t \geq 2$, let $\psi(n, t)=1$. The recurrence relation

$$
\left[\begin{array}{l}
n \\
t
\end{array}\right]=\left\{\begin{array}{l}
{\left[\begin{array}{l}
n-1 \\
t-1
\end{array}\right]+(n-1)\left[\begin{array}{c}
n-1 \\
t
\end{array}\right], \quad \text { if } n \geq 2,} \\
{\left[\begin{array}{l}
1 \\
t
\end{array}\right]=\delta_{1 t}}
\end{array}\right.
$$

ensures that $b(n, t)=\left[\begin{array}{c}n \\ t\end{array}\right]$ for all $(n, t) \in V$. Moreover, the standard constructive proof of (3.6) gives the following bijection between the paths $(n, t) \rightarrow \tau$ and the $t$-cycle permutations of $[n]$. Given a path $\omega:(n, t) \rightarrow \tau$, make the associated permutation $\sigma$ as follows.

(I) If the first edge of $\omega$ is horizontal edge $\ell$ from $(n, t)$ to $(n-1, t)$, where $1 \leq \ell \leq$ $\phi(n, t)=(n-1)$, then choose (recursively) a $t$-cycle permutation of $[n-1]$, and insert $n$ to follow $\ell$ in the cycle containing $\ell$ (so that $(n)$ will not be a 1 -cycle of $\sigma$ ).

(II) If the first edge of $\omega$ is the diagonal edge $(n, t) \rightarrow(n-1, t-1)$, choose (recursively) a $(t-1)$-cycle permutation of $[n-1]$, and adjoin $(n)$ as a 1 -cycle. 
Observe that (1.2) is the instance of (3.5) in this family. Since $\phi(n, t)=(n-1)$, we have

$$
\begin{aligned}
{\left[\begin{array}{l}
n \\
t
\end{array}\right] } & =b(n, t)=\sum_{2 \leq k_{1}<k_{2}<\cdots<k_{n-t} \leq n}\left[\prod_{i=1}^{n-t}\left(k_{i}-1\right)\right] \\
& =\sum_{2 \leq k_{1}<k_{2}<\cdots<k_{n-t} \leq n}\left(k_{1}-1\right)\left(k_{2}-1\right) \cdots\left(k_{n-t}-1\right), \\
\left(u_{i} \longleftarrow k_{i}-1\right) & =\sum_{1 \leq u_{1}<u_{2}<\cdots<u_{n-t}<n-1} u_{1} u_{2} \cdots u_{n-t} .
\end{aligned}
$$

Summand $u_{1} u_{2} \cdots u_{n-t}$ above is the number of $t$-cycle permutations $\sigma$ of $[n]$ such that the set of cycle minima of $\sigma$ is $[n] \backslash\left\{u_{1}+1, u_{2}+1, \ldots, u_{n-t}+1\right\}$.

Example 3.4. Let $V=\{(n, t): n \geq t \geq 1\}$; if $n \geq t+1 \geq 2$, let $\phi(n, t)=t$; if $n \geq t \geq 2$, let $\psi(n, t)=1$. The recurrence relation

$$
\left\{\begin{array}{l}
n \\
t
\end{array}\right\}=\left\{\begin{array}{l}
\left\{\begin{array}{c}
n-1 \\
t-1
\end{array}\right\}+t\left\{\begin{array}{c}
n-1 \\
t
\end{array}\right\}, \quad \text { if } n \geq 2 \\
\left\{\begin{array}{l}
1 \\
t
\end{array}\right\}=\delta_{1 t}
\end{array}\right.
$$

ensures that $b(n, t)=\left\{\begin{array}{l}n \\ t\end{array}\right\}$ for all $(n, t) \in V$. Moreover, the standard constructive proof of (3.8) gives a bijection between the paths $(n, t) \rightarrow \tau$ and the $t$-block partitions of $[n]$. For completeness, again, we repeat the proof here, phrasing it so as to make the bijection clear. Given a path $\omega:(n, t) \rightarrow \tau$, make the associated partition $\pi$ as follows.

(I) If the first edge of $\omega$ is horizontal edge $\ell$ from $(n, t)$ to $(n-1, t)$, where $1 \leq \ell \leq$ $\phi(n, t)=t$, then choose (recursively) a $t$-block partition of $[n-1]$, and insert $n$ in the block with the $\ell$ th smallest minimal element (so that $\{n\}$ will not be a singleton of $\pi$ ).

(II) If the first edge of $\omega$ is the edge $(n, t) \rightarrow(n-1, t-1)$, choose (recursively) a $(t-1)$ block permutation of $[n-1]$, and adjoin $\{n\}$ as a singleton.

Observe that (1.5) is the instance of (3.5) in this family. Since $\phi(n, t)=t$, we have

$$
\begin{aligned}
\left\{\begin{array}{c}
n \\
t
\end{array}\right\} & =b(n, t)=\sum_{2 \leq k_{1}<k_{2}<\cdots<k_{n-t} \leq n}\left[\prod_{i=1}^{n-t}\left(k_{i}-i\right)\right] \\
& =\sum_{2 \leq k_{1}<k_{2}<\cdots<k_{n-t} \leq n}\left(k_{1}-1\right)\left(k_{2}-2\right) \cdots\left(k_{n-t}-(n-t)\right), \\
\left(u_{i} \longleftarrow k_{i}-i\right) & =\sum_{1 \leq u_{1} \leq u_{2} \leq \cdots \leq u_{n-t} \leq t} u_{1} u_{2} \cdots u_{n-t} .
\end{aligned}
$$

In this example, summand $u_{1} u_{2} \cdots u_{n-t}$ is the number of $t$-block partitions $\pi$ of $[n]$ such that the set of block minima of $\pi$ is $[n] \backslash\left\{u_{1}+1, u_{2}+2, \ldots, u_{n-t}+n-t\right\}$. 


\section{Combinatorial identities by way of Wilf's multigraph model}

Next, we apply Theorem 3.2 in a restricted binomial family introduced by Calabi and Wilf [1].

Example 3.5. For $0 \leq t \leq n$ and for $q$, a power of a prime, define

$$
\left[\begin{array}{l}
n \\
t
\end{array}\right]_{q}:=\text { The number of } t \text {-dimensional subspaces of } \mathbf{F}_{q}^{(n)}
$$

where $\mathbf{F}_{q}$ is the finite field with $q$ elements.

These numbers satisfy the well-known recurrence

$$
\left[\begin{array}{l}
n \\
t
\end{array}\right]_{q}=\left\{\begin{array}{l}
{\left[\begin{array}{l}
n-1 \\
t-1
\end{array}\right]_{q}+q^{t}\left[\begin{array}{c}
n-1 \\
t
\end{array}\right]_{q}, \quad \text { if } n \geq 1} \\
{\left[\begin{array}{l}
0 \\
t
\end{array}\right]_{q}=\delta_{0 t} .}
\end{array}\right.
$$

In [1], Calabi and Wilf provide a constructive proof of (3.11), which we summarize here. Their proof capitalizes on the fact that for $1 \leq t \leq n$, each $t$-dimensional subspace of $\mathbf{F}_{q}^{(n)}$ is the row space of a unique $t \times n$ matrix over $\mathbf{F}_{q}$ in reduced row echelon form (rref). In the top line of (3.11), the first term is the number of $t \times n$ rref matrices for which $(t, n)$ is a pivot position, and the second term is the number of such matrices for which it is not.

This recurrent construction gives an associated restricted binomial family $G$ where the vertex set is given by $V=\{(n, t): n \geq t \geq 0\}$; if $n \geq t+1 \geq 1$, then $\phi(n, t)=q^{t}$ and if $n \geq t \geq 1$, then $\psi(n, t)=1$.

Applying (3.5) here gives, for $n \geq t+1$,

$$
\left[\begin{array}{c}
n \\
t
\end{array}\right]_{q}=\sum_{1 \leq k_{1}<k_{2} \cdots<k_{n-t} \leq n} q^{k_{1}-1} q^{k_{2}-2} \cdots q^{k_{n-t}-(n-t)}=\sum_{0 \leq u_{1} \leq \cdots \leq u_{n-t} \leq t} q^{u_{1}+u_{2}+\cdots+u_{n-t}},
$$

where summand $q^{u_{1}+u_{2}+\cdots+u_{n-t}}$ is the number of $t \times n$ rref matrices with pivots in columns $[n] \backslash\left\{u_{1}+1, u_{2}+2, \ldots, u_{n-t}+n-t\right\}$.

The Calabi/Wilf construction and formula (3.12) appear in [6].

\section{Application to unrestricted binomial families}

We now consider general binomial families, without the restriction $\psi \leq 1$. The computational form of (2.1) is obtained as much as (3.5) was, although the formula is messier. Again, consider a fixed vertex sequence from $(n, t)$ to $\tau=\left(t_{0}, t_{0}\right)$. Proceeding as before, we index this sequence by the same set $S$, but we now need to use $S$ to compute the $y$ coordinate from the $x$-coordinate for every vertex $(x, y)$ in the sequence, not just for those vertices with $x$-coordinates in $S$. The function which does this is the function

$$
f_{S}:\left[t_{0}+1, n\right] \longrightarrow\left[t_{0}+1, t\right]
$$


Table 4.1

\begin{tabular}{c|cc}
\hline$x$ & $S \cap[1, x]$ & $f_{S}(x)$ \\
\hline 1 & $\varnothing$ & $1-0=1$ \\
2 & $\{2\}$ & $2-1=1$ \\
3 & $\{2\}$ & $3-1=2$ \\
4 & $\{2,4\}$ & $4-2=2$ \\
5 & $\{2,4,5\}$ & $5-3=2$ \\
6 & $\{2,4,5\}$ & $6-3=3$ \\
7 & $\{2,4,5,7\}$ & $7-4=3$ \\
\hline
\end{tabular}

given by

$$
f_{S}(x):=x-\left|S \cap\left[t_{0}+1, x\right]\right|
$$

This is the correct function because for any vertex $(x, y)$ in the sequence, the number of horizontal edges to the left of $(x, y)$, which equals $x-y$, is given by $\left|S \cap\left[t_{0}+1, x\right]\right|$.

Again, consider the vertex sequence

$$
(0,0) \longleftarrow(1,1) \longleftarrow(2,1) \longleftarrow(3,2) \longleftarrow(4,2) \longleftarrow(5,2) \longleftarrow(6,3) \longleftarrow(7,3),
$$

indexed by the set $S=\{2,4,5,7\}$.

The function $f_{S}$ is computed in Table 4.1. Note that $f_{S}$ does correctly find the $y$ coordinate of each vertex from its $x$-coordinate. This function now gives us the analogue of (3.8) for general binomial families. The number of paths that follow the vertex sequence indexed by $S$ is given by

$$
\prod_{x \in S} \phi\left(x, f_{S}(x)\right) \prod_{x \in \bar{S}} \psi\left(x, f_{S}(x)\right)
$$

where $\bar{S}:=\left[t_{0}+1, n\right] \backslash S$. Summing over all possible subsets $S$ gives the following theorem. Theorem 4.1. Let $G$ be a binomial family, and let $(n, t) \in V(G)$, with $n \geq t+1$. Then,

$$
b(n, t)=\sum_{\substack{S \subseteq\left[t_{0}+1, n\right] \\|S|=n-t}}\left[\prod_{x \in S} \phi\left(x, f_{S}(x)\right) \prod_{x \in \bar{S}} \psi\left(x, f_{S}(x)\right)\right] .
$$

Note that $b(n, n)=\prod_{x=t_{0}}^{n} \psi(x, x)$.

Example 4.2. Formula (4.5) can be applied to a binomial family, introduced in [3], that models the following sets of trees. 
Definition 4.3. For $1 \leq t \leq(n-2)$, let $T(n, t)$ be the set of all trees on $n$ labeled vertices, where vertices $\{1, \ldots, t\}$ all have degree $\geq 2$ and vertices $\{t+1, \ldots, n\}$ are all leaves. (It is convenient to ignore the tree on one vertex and the tree on two vertices.) The triangle of numbers $\{|T(n, t)|\}$ is the sequence $A 019538$ in [5] and satisfies the recurrence relation below:

$$
|T(n, t)|= \begin{cases}1, & \text { if } t=1, \\ 0, & \text { if } t=n-1, t \geq 2, \\ t|T(n-1, t)|+t|T(n-1, t-1)|, & \text { if } 2 \leq t \leq(n-2) .\end{cases}
$$

Equation (4.6) can be established by a recurrent construction of the sets $T(n, t)$.

For $n \geq 3, T(n, 1)$ obviously contains one tree, in which vertex $n$ is adjacent to vertices $1,2, \ldots, n-1$; so let $2 \leq t \leq(n-2)$.

(I) Given a tree $\mathcal{T} \in T(n-1, t)[$ for $t \leq(n-3)]$ and an integer $j, 1 \leq j \leq t$, construct tree in $T(n, t)$ by making vertex $n$ adjacent to vertex $j$. If this is done for each choice of $\mathcal{T}$ and $j$, each tree in $T(n, t)$ for which the vertex adjacent to $n$ has degree $\geq 3$ will be constructed exactly once.

(II) Given a tree $\mathscr{T} \in T(n-1, t-1)$ and an integer $j, 1 \leq j \leq t$, construct tree in $T(n, t)$ by exchanging the labels of vertices $j$ and $t$ in $\mathscr{T}$ making vertex $n$ adjacent to the vertex now labeled $j$. If this is done for each choice of $\mathscr{T}$ and $j$, each tree in $T(n, t)$ for which the vertex adjacent to $n$ has degree $=2$ will be constructed exactly once.

We can now construct the associated binomial family; in order to do so, we must shift the graph two units to the left. Put $V=\{(n, t): n \geq t \geq 1\}$, so that $\tau=(1,1)$. For $n \geq$ $(t+1) \geq 2$, put $\phi(n, t)=t$; for $n \geq t \geq 2$, put $\psi(n, t)=t$. The trees in $T(n, t)$ are then in bijection with the paths $(n-2, t) \rightarrow(1,1)$, so that

$$
|T(n, t)|=b(n-2, t)
$$

Applying (4.5) to this binomial family and rearranging terms will now give, for $t \geq 2$,

$$
|T(n, t)|=\sum_{2 \leq k_{1}<k_{2}<\cdots<k_{t-1} \leq n-2} 2^{k_{2}-k_{1}} 3^{k_{3}-k_{2}} \cdots(t-1)^{k_{t-1}-k_{t-2}} t^{(n-1)-k_{t-1}} .
$$

The well-known Prüfer bijection between labeled trees and sequences (e.g., described in [4]) induces a bijection between $T(n, t)$ and the set $\mathscr{S}$ of surjections from $[n-2]$ onto $[t]$. There is a also a straightforward bijection

$$
\mathscr{S} \longleftrightarrow\{t \text {-block partitions of }[n-2]\} \times S_{t} .
$$

Composing these two bijections allows a variant of Example 3.4 to furnish another construction for (4.8), but the two recurrent constructions are not the same. 


\section{Derangements}

Our final example falls outside the scope of Theorems 3.2 and 4.1. A derangement of $[n]$ is a fixed-point-free permutation of $[n]$; when grouped by number of cycles, the sets of derangements of $[n]$ are modeled by a combinatorial family graph that is almost a binomial family.

Example 5.1. For $2 \leq 2 t \leq n$, let $D(n, t)$ be the set of $t$-cycle derangements of $[n]$. For all $(n, t)$ satisfying $2 \leq 2 t \leq n$, except for $(n, t)=(2,1)$, the triangle of numbers $\{|D(n, t)|\}$ satisfies the recurrence

$$
|D(n, t)|=(n-1)|D(n-1, t)|+(n-1)|D(n-2, t-1)| .
$$

Note that $\{|D(n, t)|\}$ is the sequence $A 008306$ in [5]. Now, formula (5.1) can be established by a recurrent construction analogous to that of Example 3.3. Fix $(2,1) \neq(n, t)$ such that the given inequalities hold.

(I) Given $\hat{\sigma} \in D(n-1, t)$ and integer $j, 1 \leq j \leq(n-1)$, construct $\sigma \in D(n, t)$ by inserting $n$ to follow $j$. Doing this for each choice of $\hat{\sigma}$ and $j$ will construct, exactly once, each $\sigma \in D(n, t)$ for which $n$ is not in a 2-cycle.

(II) Given $\hat{\sigma} \in D(n-2, t-1)$ and integer $j, 1 \leq j \leq(n-1)$, construct $\sigma \in D(n, t)$ as follows. (a) Make the substitution $x \leftarrow(x+1)$ for each $x \geq j$ in the domain and range of $\hat{\sigma}$ (so that $\hat{\sigma}$ becomes a $(t-1)$-cycle derangement of the set $[n-1] \backslash\{j\}$ );

(b) adjoin transposition $(n, j)$ to the transformed $\hat{\sigma}$. Doing this for each choice of $\hat{\sigma}$ and $j$ will construct, exactly once, each $\sigma \in D(n, t)$ for which $n$ is in a 2-cycle.

Note that the first term of (5.1) is zero when $n=2 t$ and the second term is zero when $t=1$.

The associated combinatorial family graph has vertex set $V=\{(n, t): 2 \leq 2 t \leq n\}$; $\tau=(2,1)$. For $n>2 t$, there are $\phi(n, t)=(n-1)$ edges $(n, t) \rightarrow(n-1, t)$; for $t \geq 2$, there are $\psi(n, t)=(n-1)$ edges $(n, t) \rightarrow(n-2, t-1)$. For any vertex $(n, t) \in V$, there are $\left(\begin{array}{c}n-t-1 \\ t-1\end{array}\right)$ vertex sequences from $(n, t)$ to $(2,1)$, one for each possible placement of the $(t-1)$ diagonal moves among the $(n-t-1)$ total moves needed.

For a given vertex sequence, let $\left\{k_{1}, k_{2}, \ldots, k_{t-1}\right\}$ be the set of $x$-coordinates of vertices to the left of the diagonal edges, where necessarily $2 \leq k_{1}<k_{2}-1<k_{3}-2<\cdots<$ $k_{t-1}-t+2 \leq(n-2)$ (i.e., where no two $k_{i}$ 's are consecutive). A little thought shows that the number of paths that follow this vertex sequence, the product of the $\phi$ and $\psi$ values along the sequence, is the product of all of the positive integers $\{2, \ldots, n-1\}$, except for $\left\{k_{1}, k_{2}, \ldots, k_{t-1}\right\}$, which must be omitted. The associated identity (for $n \geq 2 t \geq 4$ ) can thus be written as follows. This identity appears in [2],

$$
|D(n, t)|=\sum_{2 \leq k_{1}<k_{2}-1<\cdots<k_{t-1}-t+2 \leq(n-2)} \frac{(n-1) !}{k_{1} k_{2} \cdots k_{t-1}}
$$

Observe that $|D(n, 1)|=(n-1)$ !. 
We would like to thank the anonymous referee for pointing out an elegant bijection which demonstrates that summand $(n-1) ! / k_{1}, k_{2} \cdots k_{t-1}$ of $(5.2)$ is also the number of $t$-cycle derangements of $[n]$ with cycle-length vector

$$
\left(k_{1}, k_{2}-k_{1}, \ldots, k_{t-1}-k_{t-2}, n-k_{t-1}\right) .
$$

We find this fact remarkable because this gives a partition of $D(n, t)$ that is different from ours: the derangements belonging to a given vertex sequence in our family do not in fact share a common cycle-length vector.

As the examples we have considered demonstrate, recognizing an identity as an instance of Theorem 2.2 provides it with a clear interpretation and links it to other such identities. This is due to the simple unified structure that underlies all of the instances of Wilf's multigraph.

\section{References}

[1] E. Calabi and H. S. Wilf, On the sequential and random selection of subspaces over a finite field, Journal of Combinatorial Theory. Series A 22 (1977), no. 1, 107-109.

[2] R. J. Clarke and M. Sved, Derangement types, Bulletin of the Institute of Combinatorics and Its Applications 3 (1991), 21-30.

[3] P. Klingsberg, A combinatorial family of labeled trees, Journal of Algorithms 1 (1980), no. 1, 104106.

[4] A. Nijenhuis and H. S. Wilf, Combinatorial Algorithms, 2nd ed., Academic Press, New York, 1978.

[5] N. J. A. Sloane (ed.), The on-Line Encyclopedia of Integer Sequences, retrieved July 2006, from AT \&T Labs-Research. http://www.research.att.com/ njas/sequences/.

[6] R. P. Stanley, Enumerative Combinatorics. Volume I., The Wadsworth \& Brooks/Cole Mathematics Series, Wadsworth \& Brooks/Cole Advanced Books \& Software, California, 1986.

[7] H. S. Wilf, A unified setting for sequencing, ranking, and selection algorithms for combinatorial objects, Advances in Mathematics 24 (1977), no. 3, 281-291.

Theresa L. Friedman: Mesa State College, 1100 North Avenue, Grand Junction, CO 81502, USA E-mail address: tfriedman@ben.edu

Paul Klingsberg: Saint Joseph's University, 5600 City Avenue, Philadelphia, PA 19131-1395, USA

E-mail address: pklingsb@sju.edu 


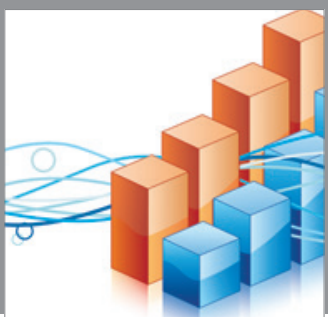

Advances in

Operations Research

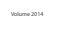

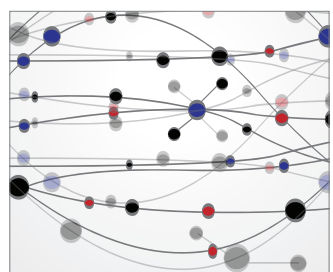

\section{The Scientific} World Journal
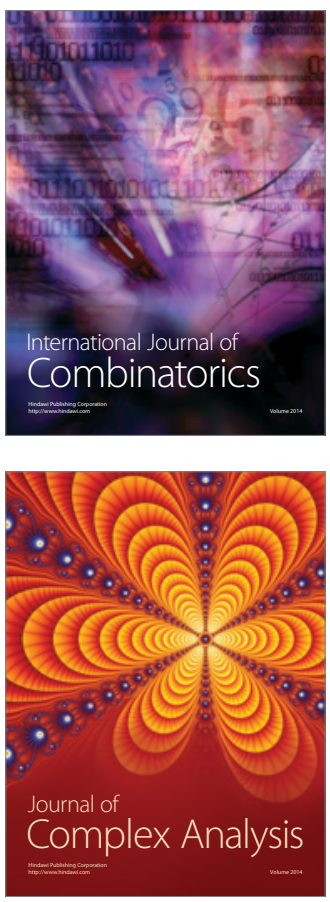

International Journal of

Mathematics and

Mathematical

Sciences
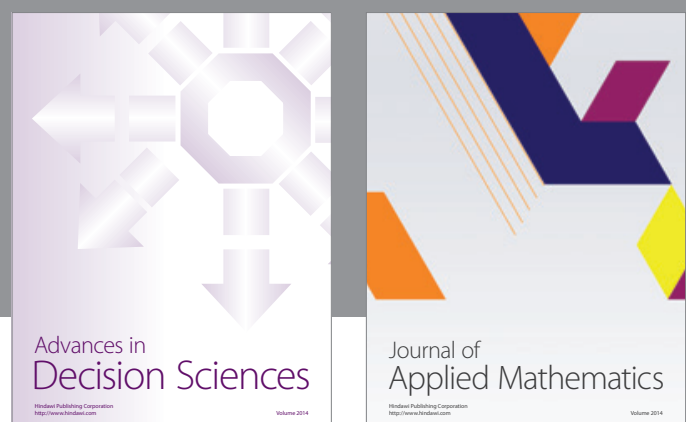

Journal of

Applied Mathematics
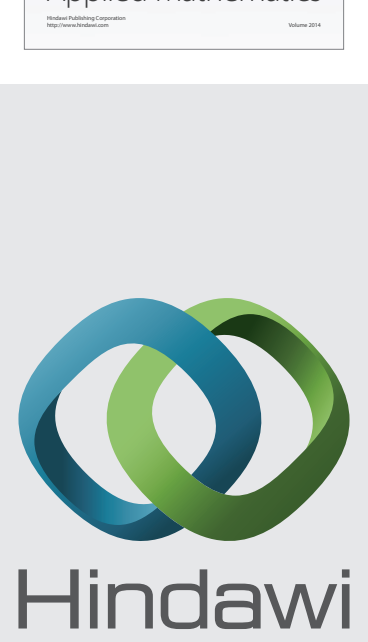

Submit your manuscripts at http://www.hindawi.com
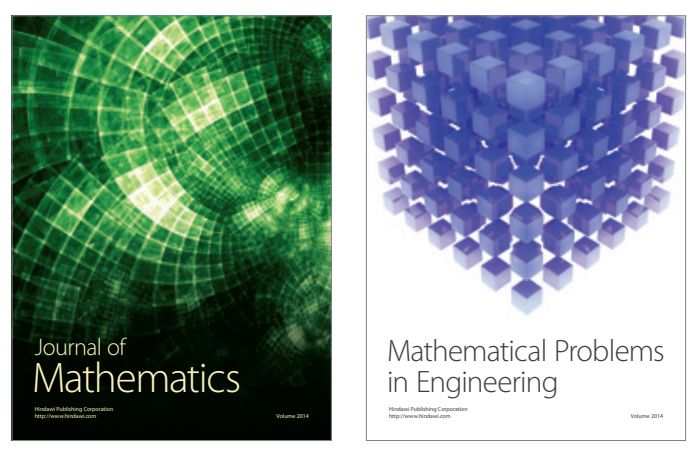

Mathematical Problems in Engineering
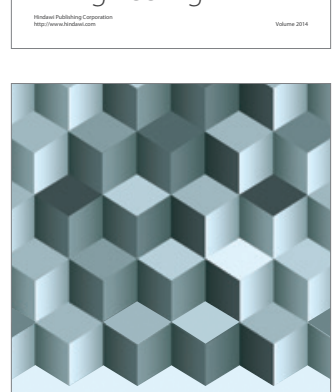

Journal of

Function Spaces
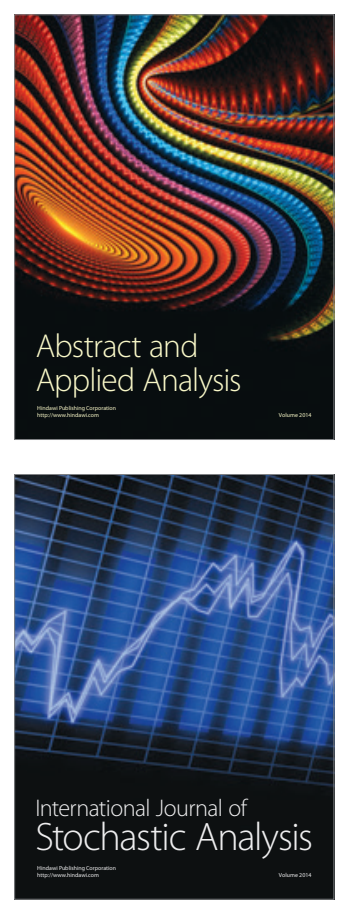

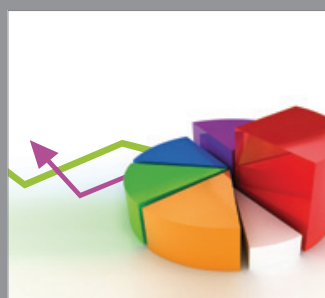

ournal of

Probability and Statistics

Promensencen
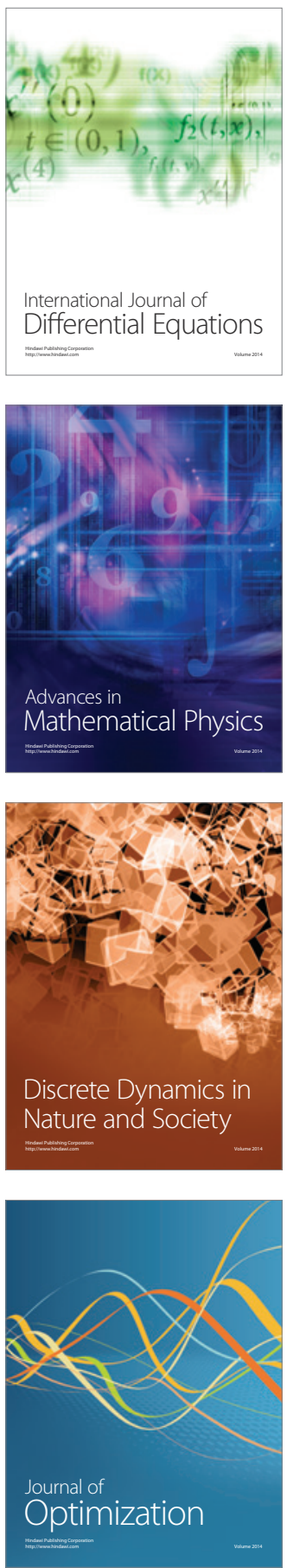